\title{
(6) OPEN ACCESS \\ Stakeholder perspectives on handovers between hospital staff and general practitioners: an evaluation through the microsystems lens
}

\section{Beryl Göbel, ${ }^{1}$ Dorien Zwart, ${ }^{2}$ Gijs Hesselink, ${ }^{3}$ Loes Pijnenborg, ${ }^{1}$ Paul Barach, ${ }^{1,5}$ Cor Kalkman, ${ }^{1,4}$ Julie K Johnson ${ }^{6}$}

\section{- Additional supplementary files are published online only. To view these files please visit the journal online (http://dx.doi.org/10. 1136/bmjqs-2012-001192). \\ ${ }^{1}$ Patient Safety Center, University Medical Centre Utrecht, Utrecht, \\ The Netherlands \\ ${ }^{2}$ Department of General Practice, Julius Center, University Medical Centre Utrecht, Utrecht, \\ The Netherlands \\ ${ }^{3}$ Scientific Institute for \\ Quality of Healthcare (IQ healthcare), Radboud University Medical Centre Nijmegen, Nijmegen, The Netherlands \\ ${ }^{4}$ Department of \\ Anesthesiology, University Medical Centre Utrecht, Utrecht, The Netherlands ${ }^{5}$ Department of Health Studies, University of Stavanger, Norway ${ }^{6}$ Centre for Clinical Governance Research, University of New South Wales, Sydney, Australia}

Correspondence to Dr Dorien Zwart, Department of General Practice, Julius Center, University Medical Centre Utrecht, P.0. Box 85500, 3508 GA, Utrecht, The Netherlands; D.Zwart@umcutrecht.nl

Accepted 2 October 2012 Published Online First 31 October 2012

\section{ABSTRACT}

Background: Much of the research on improving patient handovers has focused on enhancing communication within the hospital system, but there have been relatively few efforts aimed at addressing the challenges at the interface between the hospital and the primary care setting.

Methods: A qualitative thematic analysis using a clinical microsystems lens applied to 28 semi-structured key stakeholder interviews in the Netherlands. Data were organised into seven 'virtual' clinical microsystem datasets composed of patients, hospital-based physicians, hospital-based nurses and communitybased general practitioners.

Results: Five themes that contributed to effective or ineffective handovers emerged from our analysis:

(1) lack of adequate information; (2) healthcare professionals' availability and opportunity for personal contact; (3) feedback, teaching and protocols related to handovers; (4) information technology facilitated communication solutions; and (5) the role and responsibility of patients. Our analysis suggests that each healthcare professional attempted to provide the best care possible, but did this largely in isolation, and without the benefit of the knowledge and expertise of the other members of the microsystem.

Conclusions: The microsystem approach offers an innovative organisational construct and approach to assess the gaps in 'hospital to community' patient handovers, by viewing the hospital to the community interface as a clinical microsystem continuum. Our application of the microsystem approach confirms and extends earlier findings about the impact of barriers on the continuity and safety of patient transitions and their impact on the quality of patient care.

\section{INTRODUCTION}

Patients continue to suffer preventable harm from medical care, including serious disability and death, despite considerable improvement efforts in the past decade. ${ }^{1-5}$ Communication patterns during transitions of care remain ambiguous, non-standardised and problematic and continue to contribute to adverse outcomes. ${ }^{6}{ }^{7}$ Attention has focused on describing the extent of the problem, the underlying organisational enablers and barriers, and finding solutions to improve handovers of patient care. ${ }^{8}{ }^{9}$ Solutions to improve patient handover, communication and non-technical team and communication skills have been available worldwide. Examples include standardised communication aids such as situation, background, assessment, recommendation (SBAR) ${ }^{10}$ communication aids designed for the handover such as the iSoBar checklist, ${ }^{11}$ and TeamStepps team training. ${ }^{11} 12$

Much of this research has focused on enhancing communication within the hospital. Handovers of care in hospitals are frequent and complex due to multiple healthcare professional shift changes each day, inter-hospital referrals, and transitions of the patient from one care setting within the hospital to another (eg, from the emergency department to the operating room or the inpatient ward). There has been relatively little work on handovers between primary care and the hospital despite its frequency. For example, $10 \%$ of all patients consulting a general practitioner (GP) in the Netherlands are referred to a specialist, contributing to an estimated 120000 patient handovers a year. ${ }^{13}$

If handovers within a single facility are 'challenging', problems may be compounded in handovers across different professionals, settings and jurisdictions. ${ }^{14}$ For example, a patient's transition from a tertiary hospital back to the community may have no exchange of information or an asynchronous 
transfer of data between the hospital physician and the GP, with the patient not present while the handover occurs. The risk for the professionals to lose track of patients and their healthcare information as they move from one busy clinical setting to another is common.

We characterised and defined the relationships between the different clinical settings as a virtual clinical microsystem; 'the small, functional front-line unit that provides healthcare'. ${ }^{15}$ The microsystem comprises a group of individuals who work together, the defined setting in which they work, the patients who receive care, the processes and activities needed to accomplish the work, and the information and information technology (IT) that supports the work. Microsystems have inputs, processes and outputs (including clinical outcomes) that allow their performance to be measured and improved. ${ }^{16}$ Examples include a paediatric practice, primary healthcare practice, emergency department, intensive care unit and a surgical team. These examples assume that the care that the microsystem provides is in one discrete setting, and that transitioning to another setting involves a handover to another, different microsystem.

The handover microsystem exists at the interface between the hospital and the community. The microsystem is a powerful organisational construct for thinking about the care-giving unit that surrounds and supports the patient's needs, and helps to assess the value that the system of care is providing. Microsystems can be assessed in terms of their structure (or anatomy) and function (or physiology). Understanding the anatomy of the microsystem is related to exploration of what Nelson and colleagues call the '5Ps'-patients, professionals, purpose, processes and patterns. ${ }^{16}$ Understanding the physiology of the microsystem requires knowledge of its functional inputs and outputs. ${ }^{16}$

In thinking about handovers in the context of a microsystem, the patient, and potentially a family member, may be the only constant member of the microsystem as the patient navigates from one microsystem to another. ${ }^{17}$ Information related to the hospital discharge and follow-up care accompanies the patient, with or without IT support, and becomes part of the handover process.

The aim of this study was to apply a microsystem lens to gain insights into gaps in the handover process from the hospital to the community, and to develop recommendations for improving handovers between (local) primary and secondary care. We defined the 'handover microsystem' as the individuals (providers, patients and family members who participate in their care), activities and resources involved in the transfer of the patient from the hospital to care in the community. The handover microsystem exists at the interface between the hospital and the community. Components of this microsystem include the inpatient care team handing over the patient, the primary care team receiving the patient, and the information, technology and processes necessary to accomplish this transition.

\section{METHODS}

\section{Design}

The study was performed as part of the European FP7 Health Programme HANDOVER Research Collaborative Project. ${ }^{i}$ The HANDOVER Project studied the challenges and opportunities for improvement in patient handovers between and across facilities in six participating countries (Poland, UK, Sweden, Spain, Italy and the Netherlands) ${ }^{18-20}$ Our study was designed as a country specific sub-analysis of the larger Project focusing on results from the Netherlands.

We developed a semi-structured interview protocol and used it to interview key stakeholders of the microsystems in each country. Components of this microsystem include the inpatient care team handing over the patient, the primary care team receiving the patient, and the information, technology and processes necessary to accomplish this transition.

The protocol asked about a particular patient discharge from the hospital to the community (see online supplementary appendix A). Informed consent and other ethics requirements were met by each participating site prior to initiating the study.

\section{Settings}

We report on patients admitted to two hospitals in the Netherlands. The University Medical Centre Utrecht is an academic hospital with 1024 beds, ${ }^{21}$ and the Diakonessenhuis, a community hospital in Utrecht with 536 beds. $^{22}$ Primary care in the Utrecht region is provided by $640 \mathrm{GPs}$, who serve as gatekeepers for hospital care with no more than $5 \%$ of patients arriving at the hospital without a referral. ${ }^{23}$

\section{Participants}

We used a purposive sampling strategy to recruit participants for this study to ensure diversity of patients (eg, age, gender, diagnosis, hospital setting and wards) and care providers. Participants had to meet the following general inclusion criteria: at least 18 years old with any of the following primary diagnoses: diabetes mellitus, asthma, chronic obstructive pulmonary disease (COPD), chronic heart failure and/or patients prescribed six or more drugs, discharged to the community (home or nursing home) to be cared for under the responsibility of a primary care or community physician. Patients were recruited directly and consecutively at hospital 
discharge. We excluded patients referred to other care units within the hospital prior to discharge and patients discharged to another country.

Hospital care team members included the main providers (one for each profession) of the patients recruited for the study, and were selected from the general medical and surgical wards where the patients were hospitalised. The primary care team members worked in the communities in which the patients were discharged.

\section{Data collection}

We conducted semi-structured individual interviews using interview guides that were developed by the HANDOVER Research Consortium (see online supplementary appendix A). ${ }^{24}$ We aimed for a complete set of interviews that represented the full make-up of the microsystem: a patient, a hospital physician, a hospital nurse and a community-based GP. The interview questions were tailored to the interviewees' ability to answer and the goals of the study. The questions were designed to ask about general handover communication, perceptions of patient transitions the stakeholder had recently experienced. In addition, the cultural probes were designed to determine the characteristics of the communication between professionals in primary care and those in the hospital, and between the professionals and the patient. All interviews were audio-taped and then transcribed.

\section{Data analysis}

Of a total of 84 interviews, we identified 28 interview transcripts that constituted seven complete microsystems. Two researchers (BG and DZ) conducted a qualitative thematic analysis on each of the Dutch transcripts according to the 'social science queries' technique, whereby themes emerge when studying the transcripts regarding 'setting and context, perspectives of the informants and informants' views of thinking about people, objects, processes, activities, events and relationships'. ${ }^{25}$ Any disagreements were discussed until consensus was reached in all cases. The results from the analytical process were translated into English for compilation and analysis.

\section{RESULTS}

\section{Patients}

We interviewed seven patients (two women, two men), with a median age of 67 years. The patients were selected according to the inclusion criteria and interviewed upon discharge from the hospital. Three patients were admitted for general surgery: elective aneurysm repair, emergency hip fracture and admission to general surgical ward to rule out appendicitis; one patient was readmitted for ischaemic complications after an earlier peripheral arterial bypass operation; there were two patients with COPD and one patient with diabetes mellitus. Six patients were discharged from the hospital to their homes and one patient was discharged to a rehabilitation facility.

\section{Care providers}

The 21 healthcare professionals (table 1) were interviewed at varying time periods after the patient's discharge from the hospital. The professional medical experience of interviewees varied from being a physician in training to having 26 years of experience.

\section{Analysis}

The analysis of the seven microsystem datasets suggests that in all the microsystems ineffective handovers resulted in patients being discharged without sufficient, accurate, understandable information (for both the patient and professional) leading to suboptimal care and follow-up, and which could have resulted in harm. Miscommunications occurred for a variety of reasons, including individual professional factors, organisational and technical factors, social context and patient behaviour. ${ }^{26}$

Five themes emerged from our analysis related to effective or ineffective handovers: (1) lack of adequate information; (2) healthcare professionals' availability and opportunity for personal contact; (3) feedback, teaching and protocols related to handovers; (4) IT facilitated communication solutions; and, (5) the role and responsibility of the patient. Two of the seven cases are presented below to illustrate these themes (figure 1 and 2) and to highlight the differences in the perspectives of the various stakeholders.

\section{Theme 1:Lack of adequate information}

The GP in the first case study reported she could not reach the patient after receiving a letter that the patient had been discharged home (quote 1), when in reality, the patient had been transferred to a nursing home (quote 20). The GP phoned the patient several times at home and, not being able to reach him, assumed he was staying at his daughter's house. The GP later learned (quote 3) that her patient had been transferred to a nursing home for rehabilitation. In addition, the GP believed her patient had been transferred to a certain nursing home, while the interview with the nurse revealed that the patient had been transferred to a different nursing home (quotes 3, 20 and 26). 
Table 1 Characteristics of participating healthcare professionals

\begin{tabular}{lllll}
\hline & All care providers & Hospital physicians & Hospital nurses & General practitioners \\
\hline Female & 13 & 4 & 7 & 2 \\
Male & 8 & 3 & 0 & 5 \\
Years in profession (range) & & $3(0-10)$ & $8(3-13)$ & $21(13-26)$ \\
Total & 21 & 7 & 7 & 7 \\
\hline
\end{tabular}

\section{Theme 2:Healthcare professionals' availability} and opportunity for personal contact

The GP informed the interviewer that she would like to be contacted for updates on her patient more often by a hospital physician, but also admitted she was not sure if she put the phone number reserved for hospital physicians on the patients' referral letter (quote 2). The hospital-based physician indicated that GPs are hard to get hold of due to their differing schedules and ambiguous contact information (quote 9).

All three healthcare providers expressed the importance of personal contact to establish and/or enhance the professional relationship with their colleagues (quotes 2 , $6,7,9,17$ and 18). However, the analysis revealed that none of them initiated the first contact (quotes 4, 7 and 16), and that communication happens mostly in written form (quotes 5, 6 and 18), and personal contact appeared to be the exception rather than the rule. Healthcare professionals in all seven microsystems indicated that even when patients might be at risk at time of discharge, an attempt to establish personal contact with the 'other caregiver' in the microsystem was rare.

The stakeholders agreed that communication between the hospital and primary care is essential, but currently mostly lacking. The reported main barrier was that proper communication takes time and that there are too many individuals involved (quote 11).

The second case study (figure 2), presents a similar dynamic of miscommunication, with both the GP and nurse practitioner believing in the value of personal (phone) contact (quotes 4 and 16), but actual practice suggesting problems with accessibility.

\section{Theme 3:Feedback, teaching and protocols related to handovers}

In the first case study, the physician assistant and nurse practitioner were pleased with the manner in which they

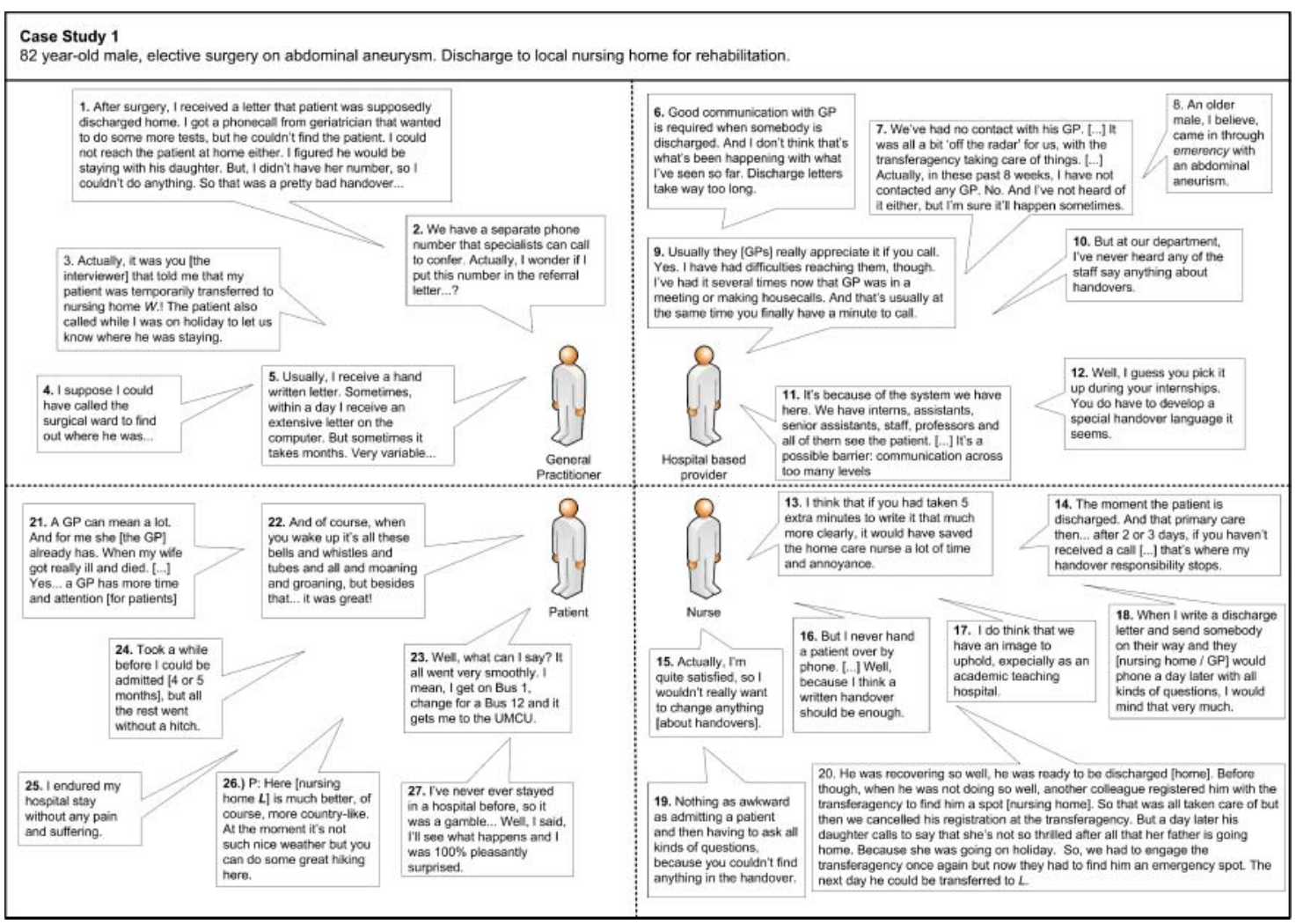

Figure 1 First case study. 


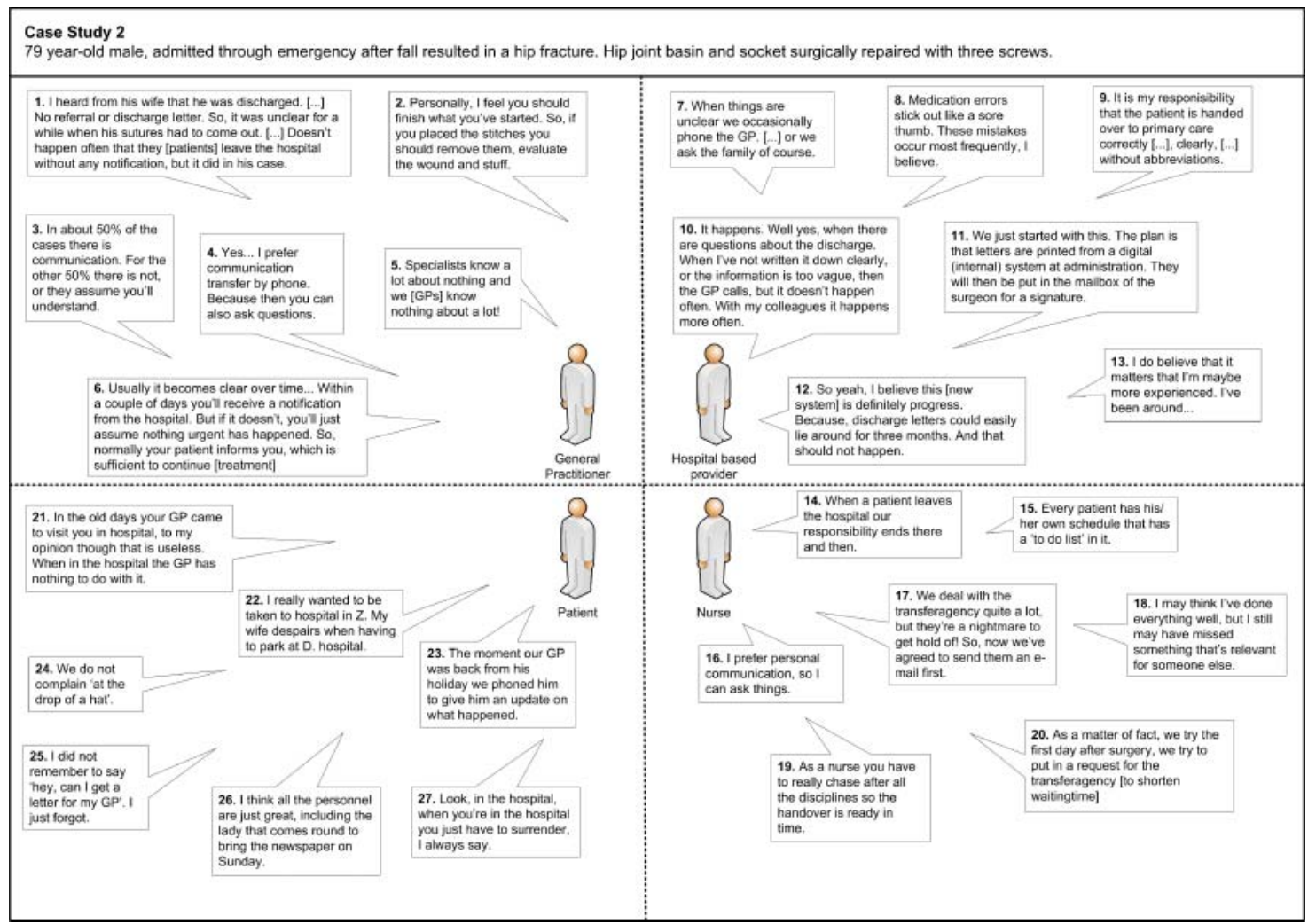

Figure 2 Second case study.

handed over the patient (quote 9, 12 and 19), but were unaware their approach had malfunctioned. The GP received neither a discharge letter nor specific instructions for removing the patient's sutures (quote 1). The professionals remarked that managing patient handovers does not seem to be part of their training or based on a common protocol $(10,12$ and 13). While the nurse was confident she did a good job transitioning the patient through the transfer agency (which supports hospital staff with making the discharge arrangements), she acknowledged she rarely received feedback (quote 18). She also indicated she feels responsible for ensuring that the handover is made in a timely way (quote 19).

\section{Theme 4Information technology (IT) facilitated communication solutions}

The physician assistant in the second case study mentioned a new electronic communication system that sends digital letters on the day of discharge (quote 11 and 12). He admitted that there are limitations to this new approach: "the system sends the letters to administration who print it, because it has to be signed by the responsible physician", which increases turnaround time. A general complaint about IT-solutions by professionals from all seven microsystems was the variety of systems, inadequate training on their use, lack of IT support personnel and the extra time needed to complete the electronic forms.

\section{Theme 5:Patients' Role and Responsibility}

The role and responsibility of patients in their handover is not clear to the healthcare professionals involved. This dissonance is illustrated in both case studies and mirrors the findings of Flink et $a l^{23}$ In the first case study, even with inconsistencies in the communication and process failures, the patient was satisfied with the experience and did not seem to realise that anything had gone amiss. The patient thought highly of his GP (quote 21), thought his hospital stay was "great" (quote 22), liked the location of his rehabilitation (quote 26), and regarded the public transport to the hospital as 'smooth' with only two bus changes (quote 23).

By contrast, the patient in the second case study viewed himself as an integral part of the handover, and felt responsible for the miscommunication: "I forgot to ask for a letter [from my discharge physician] for my GP" (quote 25). And while the patient is clearly engaged with his GP (quote 23), in his mind the primary care and hospital care were disconnected and he questioned the role of the GP during the hospital stay, "to my opinion K in the hospital the GP has nothing to do with it" (quote 21). The patient also viewed his role during 
the inpatient course as disempowered "in the hospital, you just have to surrender yourself" (quote 27), and his main concern related to his ability to influence which emergency department would admit him (quote 22).

\section{DISCUSSION}

We elicited and analysed the actions of microsystem stakeholders involved in patient handovers through a $360^{\circ}$ 'microsystem lens'. This innovative approach allowed us to consider the inputs and reflections of key stakeholders as well as the context of the handover. Our findings demonstrate that each healthcare professional attempted to provide the best care possible, but largely did so in isolation, and without the benefit of the knowledge and input of the other members of their respective microsystem.

Our findings highlight the risks of a fragmented healthcare system in which there are often too many people involved in the handover process, each with ambiguously defined responsibilities. The healthcare professionals indicated that they work without clear and shared protocols and that IT tools to support handover communication are rarely used, largely due to a lack of interoperability between systems from different vendors. Despite the stakeholders' efforts and best intentions, handovers were inefficient and demonstrated flaws and process failures. Although many respondents talked about potential improvements, there was little evidence of efforts to change the current processes. Surprisingly, the interviewees felt powerless about changing or improving the situation.

Our findings align with the notion about the barriers and facilitators for effective handover found in other studies. Berendsen et al investigated the cooperation between GPs and specialists in the Netherlands, ${ }^{27}$ and reported that a lack of personal contact between providers was perceived as a barrier to fruitful collaboration between GPs and hospital physicians.

Solutions proposed such as 'make technological changes (simplify and standardise), use information- (or evidence-) based strategies, take human factors into consideration (eg, communication and training) and appoint a leader (coordinator)' seem straightforward, but they are highly variable in their success and impact, and require resources that are not available. ${ }^{28-30}$ To our knowledge, existing solutions for optimising patient handovers in complex systems have not been implemented fully in most settings. In addition, there is a paucity of research describing the impact of educational interventions to improve handover and to assess their effectiveness. Some evidence exists to demonstrate that skills can be transferred to the workplace, ${ }^{23}$ but no studies were found to demonstrate that interventions sustainably improve patient safety.

\section{Interpretation of our findings}

While we worked with a small sample of 21 healthcare provider interviews, comments on the lack of standardisation, coordination and training for handover were consistent across the group; interviewees were quite frustrated with low degree of coordination within the system. The second case study illustrates an example in which a recently implemented technological tool to facilitate handover was not optimally utilised and was unable to guarantee a successful patient transition from the hospital to the patient's home. Other studies have also found that the most effective measures to support GP and specialist cooperation is to allow for easy telephone access (for patients and healthcare professionals), increase the timeliness of discharge letters, and create feedback between professionals through frequent meetings. ${ }^{27} 31$

The handovers we assessed were suboptimal because stakeholders largely work in isolation, leading to ineffective care and potential harm. Healthcare professionals seem to have both a physical and a psychological difficulty contacting and communicating with one another (figure 1, quote 7). Care providers struggle on their own (figure 1, quote 4), and will even reach out to patients or family members to help resolve ambiguous issues, but hesitate to discuss ambiguous issues with their colleagues (figure 1, quote 19).

A possible explanation may be an inability to grasp the interdependencies of the system, in that 'humans tend to focus on local aspects and do not easily grasp systemic and non-linear relationships'. ${ }^{32}$ In other words, healthcare professionals can be proficient in their own clinical domain (ie, internal medicine, paediatrics, GP practice, etc), but may not be equipped nor trained to appreciate their impact on the larger system and its impact on patient outcomes. This is expressed eloquently by the nurse in case study 2: "When a patient leaves the hospital, our responsibility ends there and then." At the same time, this lack of discharge coordination is puzzling given the high performance of the Dutch primary care gatekeeper system, in which less than $5 \%$ of patients are admitted to the hospital without a GP referral.

A further unresolved challenge in explaining the ineffective handovers is the lack of consensus about the different levels of communication that are required between healthcare providers. ${ }^{33}$ GPs reported they would like to receive an high level overview of the patient's hospital stay, but did not feel a need to know the detailed data such as blood pressure or laboratory results at the time of admission, nor information on the 
course of the hospitalisation. The GP (and the patient) does need a summary of the hospital stay and what should or may happen next, could benefit from the anticipatory guidance, and how best to respond if these symptoms or outcomes change ('what if?'). Understanding these mindsets may facilitate anticipatory management- 'if this happens, then do that'. The GP sender also needs to be continuously aware of the receiver's focus of interest (ie, relevant knowledge for a cardiologist may not be relevant for a GP). In the second case study the nurse remarks: "I may think I've done everything well, but I still may have missed something that's relevant for someone else" (quote 18).

A third interpretation is that handovers are not yet an integral part of the required education of healthcare professionals. Handover competencies are rarely explicitly taught in undergraduate and graduate healthcare professions training, and clinicians mostly learn these skills by 'being around' and immersed in the clinical effort (figure 2, quote 13). Furthermore, while most healthcare professionals regarded handovers as an essential part of the patient care process, they were simultaneously seen as an administrative burden that detracts them from their other patient care duties. One example of this de-prioritisation and delegation of handover tasks is the observation that when we attempted to interview the lead physician for each patient, in four out of the seven cases, we were 'assigned' one of his or her trainees, interns, or physician assistants. At the same time, these junior healthcare professionals were more directly involved in the care process and knew the patient's clinical issues. In the three cases where we did get to speak to the responsible physician, only one physician had actually been present at the patient's discharge.

\section{Solutions}

Structured feedback following the handover might improve the personal contact and likely, the quality of patient handovers. None of the professionals interviewed received feedback on their handovers, while all indicated they would welcome structured feedback. Other solutions that emerged from our interviews included improving non-technical skills and teamwork, ${ }^{34}$ crossover training and multi-disciplinary training. ${ }^{35}$ Standardised formats (eg, checklists), digital patient files and other IT solutions were also viewed as helpful and are increasingly embedded in daily practice. Fully using them would require overcoming the connectivity issues that currently appear to exist between individual legacy systems. Finally, improving the handover process requires defining the role of the patient in this process, and designing practical ways to actively involve patients in their handovers.
There are several limitations to this study. The hospital-based physicians and the primary care givers were interviewed several days to weeks after their patient was discharged. This may have induced error or recall bias. ${ }^{36}$ Interviewees may also have replied with socially desirable answers (social desirability response bias). ${ }^{37}$ The study is based on a small sample size of 28 interviews and did not include other community-based providers, such as nurses and pharmacists, and our data reflects the findings for two hospitals in the Netherlands, and it may not be possible to generalise to other institutions or nations. We believe our findings are illustrative of handovers of patients from the hospital settings to the community, and results of the HANDOVER Project found similar barriers to effective handovers across the five healthcare systems studied. ${ }^{18}$

\section{CONCLUSIONS}

Our study offers an innovative approach to assessing and addressing the gaps between current handover practices from the hospital to the community by viewing this interface as a virtual microsystem. The analysis indicates that although the healthcare providers we studied do their best, the result can be ineffective because they work in isolation, not taking advantage of the virtual microsystem that exists between these care settings. Improvement will require building knowledge of the anatomy and physiology of the microsystem and the dynamic inputs, processes, outputs and feedback loops within the system.

Further research is needed to assess the impact and cost of implemented solutions, such as locally customised, agreed feedback mechanisms, and/or other nontechnical solutions, such as multi-disciplinary virtual and face to face education and meetings, and crossover training between primary and secondary healthcare providers. Both quantitative and qualitative 'pre/post' designs are needed to quantify the added value of such structural changes in training and the organisation of crossdisciplinary teamwork. ${ }^{38}$

Acknowledgements We thank all the members of the European HANDOVER Research Collaborative for their contribution: Avedis Donabedian University Institute Autonomous University of Barcelona (Spain), University of Birmingham (UK), National Centre for Quality Assessment in Healthcare (Krakow, Poland), Azienda Sanitaria Firenze (Italy), Radboud University Medical Centre (The Netherlands), Educational Technology Expertise Center (Open University, The Netherlands) and Karolinska Universitetssjukhuset (Sweden). We also thank the healthcare providers, patients and their relatives who participated in this study.

Contributors Study conception and design: BG, DZ, CK, LP, JKJ; Acquisition of data: GH, LP; Data analysis and interpretation: BG, DZ; Drafting and/or revising the article: $B G, D Z, G H, L P, P B, C K$, JKJ.

Funding This study was supported by a grant from the European Union, the Framework Programme of the European Commission

(FP7-HEALTH-F2-2008-223409). The study sponsor had no role in the study 
design; collection, analysis, and interpretation of the data, or in the writing of the article and decision to submit the article for publication.

Competing interests None.

Ethics approval Each hospital site approved the study beforehand (UMC Urecht and Diakonessenhuis Utrecht).

Provenance and peer review Not commissioned; externally peer reviewed.

\section{REFERENCES}

1. de Vries E, Ramrattan M, Smorenburg S, et al. The incidence and nature of in-hospital adverse events: a systematic review. Qual Saf Health Care 2008;17:216-23.

2. Wagner C, de Bruijne M. Onbedoelde schade in Nederlandse Ziekenhuizen. EMGO Instituut en NIVEL, 2007.

3. Zegers M, de Bruijne MC, Wagner C, et al. Adverse events and potentially preventable deaths in Dutch hospitals: results of a retrospective patient record review study. Qual Saf Health Care 2009;18:297-302.

4. Wachter RM. The end of the beginning: patient safety five years after 'to err is human'. Health Aff (Millwood) 2004;(Suppl Web Exclusives):W4-534-45.

5. Wachter RM. Patient safety at ten: unmistakable progress, troubling gaps. Health Aff (Millwood) 2010;29:165-73.

6. Johnson JK, Arora VM. Improving clinical handovers: creating local solutions for a global problem. Qual Saf Health Care 2009;18:244-5.

7. World Health Organization. Patient Safety: Action on Patient Safety: High 5s, 2007.

8. Forster AJ, Clark HD, Menard A, et al. Adverse events among medical patients after discharge from hospital. CMAJ 2004;170:345-9.

9. Forster AJ, Murff HJ, Peterson JF, et al. The incidence and severity of adverse events affecting patients after discharge from the hospital. Ann Intern Med 2003;138:161-7.

10. Institute for Healthcare Improvement. SBAR technique for communication: a situational briefing model. Cambridge, MA Institute for Healthcare Improvement, 2012.

11. Mayer C, Cluff L, Lin W, et al. Evaluating effects to optimize TeamSTEPPS implementation in surgical and pediatric intensive care units. Jt Comm J Qual Saf 2011;37:365-274.

12. Baker D, Salas E, King $H$, et al. The role of teamwork in the professional education of physicians: current status and assessment recommendations. Jt Comm J Qual Patient Saf 2005;31:185-202.

13. Cardol M, van Dijk L, de Jong J, et al. Tweede nationale studie naar ziekten en verrichtingen in de huisartspraktijk: Huisartsenzorg: wat doet de poortwachter? (Second Dutch national survey in general practice: General practice care: how is the gatekeeper's role?). Utrecht/Bilthoven: Nivel/RIVM, 2004.

14. Johnson JK, Barach P. Patient care handovers: what will it take to ensure quality and safety during times of transition? Med J Aust 2009;190(11 Suppl):S110-12.

15. Nelson E, Batalden P, Huber T, et al. Microsystems in health care: part 1. Learning from high-performing front-line clinical units. $J t$ Comm J Qual Improv 2002;28.

16. Nelson E, Batalden P, Godfrey M, et al. Value by design: developing clinical microsystems to achieve organizational excellence. New Jersey: Jossey-Bass, 2011.

17. Mohr J, Batalden $P$, Barach $P$. Integrating patient safety into the clinical microsystem. Qual Saf Health Care December 2004; 13(Suppl 2):ii34-8.
18. Barach $\mathrm{P}$, Gademan $\mathrm{P}$, Kalkman $\mathrm{C}$, et al. Identify basic elements of effective communication. Utrecht, Netherlands: The European HANDOVER Research Collaborative, 2010.

19. Lilford R, Chilton P, Hemming K, et al. Report on the likely cost of the various prototype interventions based on a model of the likely costs. Utrecht, Netherlands: The European HANDOVER Research Collaborative., 2010

20. The European HANDOVER Research Collaborative. HANDOVER.

21. UMC Utrecht. Jaardocument 2010 Universitair Medisch Centrum Utrecht. Utrecht, The Netherlands, 2010.

22. Diakonessenhuis Annual Report 2010, 2010.

23. Enthoven AC, van de Ven WP. Going Dutch-managed-competition health insurance in the Netherlands. $N$ Engl $J$ Med 2007;357:2421-3

24. Hansagi $\mathrm{H}$, Olsson M, Bergenbrant S, et al. Report on the barriers and facilitators to effective handovers in the social, linguistic and technological contexts in different European settings. Utrecht, Netherlands: The European HANDOVER Research Collaborative, 2010.

25. Bogden R, Biklen S. Qualitative research for education: an introduction to theory and methods. Boston: Allyn and Bacon Inc., 1982.

26. Hesselink G, Zegers M, Schoonhoven L, et al. Mechanisms and processes responsible for implementing results and addressing variation within target groups. Utrecht: UMC Utrecht, 2011.

27. Berendsen AJ, Kuiken A, Benneker WH, et al. How do general practitioners and specialists value their mutual communication? A survey. BMC Health Serv Res 2009;9:143.

28. Wilson R, Runciman W, Gibberd R, et al. The quality in Australia health care study. Med J Aust 1995(163):458-76.

29. Baker $\mathrm{G}$, Norton $\mathrm{P}$, Flintoft V, et al. The Canadian adverse events study: the incidence of adverse events among hospital patients in Canada. CMAJ 2004;170:1678-86.

30. Expert Group on Learning from Adverse Events in the NHS. An organisation with a memory. London: Stationery Office, 2000.

31. Kripalani S, LeFevre F, Phillips CO, et al. Deficits in communication and information transfer between hospital-based and primary care physicians: implications for patient safety and continuity of care. JAMA 2007;297:831-41.

32. Sterman J. Modeling managerial behavior: misperceptions of feedback in a dynamic decision making experiment. Manage Sci 1989:35321-39.

33. Weggeman M. Kennismanagement: Inrichting En Besturing Van Kennisintensieve Organisaties, 4th edition, 2001, Scriptum Books, Schiedam

34. Schraagen JM, Schouten T, Smit M, et al. A prospective study of paediatric cardiac surgical microsystems: assessing the relationships between non-routine events, teamwork and patient outcomes. BMJ Qual Saf 2011;20:599-603.

35. Baker D, Gustafson S, Beaubien J, et al. Medical team training programs in health care. Advances in patient safety: from research to implementation: vol 4, programs, tools, and products. Rockville, MD: Agency for Healthcare Research and Quality, 2005.

36. Biemer P, Groves R, Lyberg R, et al. Measurement errors in surveys. Hoboken, New Jersey: John Wiley \& Sons, Inc, 2004.

37. Paulhus D. Measurement and control of response bias. In: Robinson J, Shaver P, Wrightsman L, eds. Measures of personality and social psychological attitudes. Oxford, UK: Elsevier, 1991::17-59.

38. Lilford $\mathrm{R}$, Chilton $\mathrm{P}$, Hemming $\mathrm{K}$, et al. Evaluating policy \& service interventions: a methodological classification. Br Med J 2010;341: c4413.

i www.handover.eu 